\title{
Four-Chamber 3-D Statistical Shape Model from Cardiac Short-Axis and Long-Axis MR Images
}

\author{
J. Lötjönen ${ }^{1}$, J. Koikkalainen ${ }^{2}$, D. Smutek ${ }^{1}$, S. Kivistö ${ }^{3}$, and K. Lauerma ${ }^{3}$ \\ 1 VTT Information Technology, P.O.B. 1206, FIN-33101 Tampere, Finland \\ Jyrki.Lotjonen@vtt.fi \\ 2 Laboratory of Biomedical Engineering, Helsinki University of Technology \\ P.O.B. 2200, FIN-02015 HUT, Finland \\ 3 Department of Radiology, Helsinki University Central Hospital \\ P.O.B. 340, FIN-00029 HUS, Finland
}

\begin{abstract}
We describe a new statistical atlas of the heart consisting of atrias, ventricles and epicardium. The atlas was constructed by combining information on standard short-axis and long-axis cardiac MR images. The variablity of the shape was modeled in the atlas by a statistical deformation model and by nonparametric probability distributions. The atlas has been built from 16 subjects.
\end{abstract}

\section{Introduction}

Cardiac imaging has an established role in diagnosis of cardiac diseases. Currently modern imaging modalities produce detailed anatomic and functional information of the heart. Automated analysis of huge amounts of image data is usually a pre-requisite for comprehensive quantitative analysis of cardiac images [1].

Many different types of atlases as well as different techniques to generate them have been published for the brain [2,34,5] and for the heart [67]. In many atlases, the shape variability has been also modeled. The most popular approach for modeling the shape changes is the active shape model (ASM) [8] while the corresponding technique for modeling the appearance is the active appearance model [9]. ASM defines a mean model and its typical deformation modes on the basis of a training set. The deformation modes are the eigenvectors of the covariance matrix determined for point-to-point correspondence between the different models in the training set. Another approach to model the spatial variability of 3-D points is parametric Gaussian shaped normal distributions in [23].

This paper concentrates on the shape modeling of the heart. To our knowledge statistical models have been built thus far only from ventricles and epicardium, and only short-axis (SA) images have been utilized. Since the slice thickness is normally several times larger than the pixel size in cardiac MR images, the resolution is poor in the direction orthogonal to the slice. Therefore, the accurate localization of the ventricles in the basal and apical levels has been difficult from SA images. In practice, the ventricle models are simply cutted by a plane at some basal level. This certainly imposes some inaccuracies to quantitative measures computed from the heart.

In this paper both short-axis and long-axis (LA) images were used to construct the geometry of ventricles. LA images provide comprehensive information on the modeling 


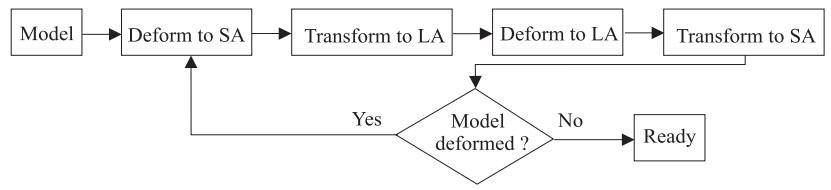

Fig. 1. Segmentation flow-chart.

the basal and apical levels more accurately. In addition, atrias were also included in the model. Mean shape and gray-scale models were constructed. Also the variablity of the shape was modeled by statistical deformation models [10], closely related to ASMs, as well as by a new non-parametric spatial probability distributions approach.

\section{Methods}

\subsection{Materials}

Sixteen healthy adult subjects were scanned twice using a 1.5 T Siemens Magnetom Vision imager (Siemens, Erlangen, Germany) at the Department of Radiology in Helsinki University Central Hospital (HUCH). During each imaging session SA and LA cine images were aquired using a standard imaging protocol adopted for cardiac patients in HUCH: SA images contained ventricles from valve level until the level where the apex was still visible, and LA images contained atrias and ventricles. In practice, a slight tilt compared to the standard LA view is used in HUCH in order to get the mitral and tricuspidal valves to the same image slice. The pixel size was $1.4 \times 1.4 \mathrm{~mm}$ for the SA and LA images. The slice thickness was $7 \mathrm{~mm}$ for the SA images and $7 \mathrm{~mm}$ for the LA images. The corresponding values for the slice separation were $15 \mathrm{~mm}$ and $10 \mathrm{~mm}$. The number of slices varied from 4 to 8 depending on the size of the heart. From 22 to 30 time points were taken for each section with a repetition time of $33 \mathrm{msec}$.

\subsection{Segmentation}

Atrias, ventricles and epicardium were manually segmented from the images. A software tool, designed for interactively making 3-D deformations, was used. With it a triangulated surface model was registered manually and non-rigidly to edges in the SA and LA image volumes.

The SA and LA volumes were interpolated isotropic using a shape-based interpolation algorithm before segmentation [11]. Thereafter, the prior model was manually deformed to a SA volume. After finishing the deformation in the SA volume, the deformed model was transformed to the co-ordinate system of the corresponding LA volume and the matching was continued in the LA volume. The deformed model was transferred iteratively between the volumes until no deformations were needed. The procedure used is shown in Fig. 1 .

The rigid transformation, $\mathbf{f}$, used to transform the model from the SA volume to the LA volume ( $\mathbf{f}^{-1}$ from LA to SA) was extracted from image file headers which contained 


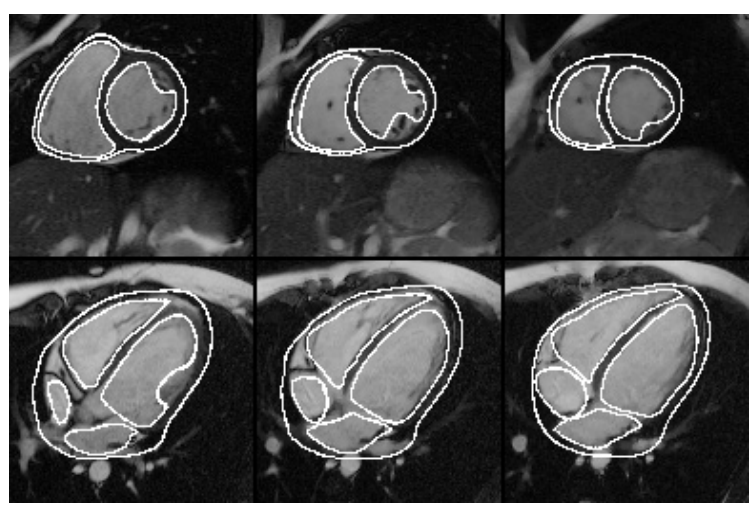

Fig. 2. A segmentation result for one subject shown on a few slices. A triangulated model has been superimposed on the SA volume (the top row) and on the LA volume (the bottom row). The rigid transformation $\mathbf{f}$ was used to transfer the model to the co-ordinate system of the LA volume.

the orientation and the position of the slices relative to the patient's anatomy. In practice, eight points were chosen from the corners of the SA volume (cube) and their co-ordinates in the LA volume were defined based on the header information. Thereafter, a markerbased registration approach, i.e. the approach that minimized the distance between the corresponding points in the SA and LA volumes, was used to define translation and rotation.

Because all images were obtained at breath hold, we detected differences in the position of the heart between the breath holds. If the differences were not corrected, the transformation derived from the image headers would not not produce good results. In this study, movements were detected and corrected separately in each image volume. Image sets orthogonal to original slice orientation were generated and the slices were moved manually, if any abnormal discontinuities were detected in structures. However, because of a separate clinical study, each subject was imaged twice, and the acquisition containing less movement-based errors was selected for the model construction. In practice, the slice misalignements were corrected only from three subjects, and the other 13 were considered to contain no movements.

A segmentation result for one subject is shown in Fig. 2]

\subsection{Construction of Mean Model}

In the construction of the mean model, the approach proposed in [10] was followed. The procedure is shortly summarized.

Instead of registering non-rigidly gray-scale volumes, as done in the original method, segmented data was utilized. First, intensity volumes were created from surface data. In these intensity volumes, each object was represented using one gray-level value. One volume, volume $V_{0}$, out of $N$ volumes was chosen to be a reference model. Then, the other volumes $V_{1}, V_{2}, . ., V_{N-1}$ were registered to the reference using translation, rotation and isotropic scaling. The gradient minimization technique was used to maximize the normalized mutual information [12] between the volumes. 
Next, the reference volume, $V_{0}$, was non-rigidly registered to the coarsely registered volumes $V_{1}, V_{2}, \ldots, V_{N-1}$ using a registration tool based on the normalized mutual information and deformation spheres [13]. The program outputs a volumetric transformation $\mathbf{T}: x \mapsto x^{\prime}$. The transformation vectors of the reference data points to volume $V_{i}$ are denoted by $\mathbf{d}_{i}$, where $i \in \Omega$ and $\Omega=\{1,2, . ., N-1\}$.

The mean model was computed by applying the mean of the transformations $\mathbf{d}_{i}$ to the reference model:

$$
\overline{\mathbf{d}}=\sum_{i \in \Omega} \mathbf{d}_{i} .
$$

The transformations from the mean model to the volumes $V_{i}$ can be computed simply $\mathbf{d}_{i}^{\prime}=\mathbf{d}_{i}-\overline{\mathbf{d}}$.

The mean model generated is mean only in the sense of shape not appearance, i.e. gray-scale. Although the emphasis in this paper is to model the variability of shape not appearance, a mean model also for appearance was computed by averaging the gray-scale volumes $V_{i}$ voxel-by-voxel after applying the transformation $-\mathbf{d}_{i}^{\prime}$.

\subsection{Modeling the Variability of Shape}

The variability in the shape was modeled in two ways: statistical deformation models (SDM) [10] and probability distributions of landmark points.

Statistical Deformation Models. In active shape models [8], typical deformation modes are searched for the surface points. SDM defines the modes for the 3-D transformation $\mathbf{T}$ itself. In both cases, the change in model's shape is written as follows:

$$
\mathbf{d}=\overline{\mathbf{d}}+\Phi \mathbf{b},
$$

where the matrix $\Phi$ consists of eigenvectors $\phi_{k}$ of the covariance matrix defined for the vectors $\mathbf{d}_{i}$, i.e. the deformation modes, and $\mathbf{b}$ is a vector containing weights for the eigenvectors.

Probability Distributions. In most cases, the assumption of the ellipsoid-shaped variability of landmarks is applicable, as assumed in parametric Gaussian shaped normal distributions [23]. However, if, for example, the twisting motion of the heart was modeled, a Gaussian distribution would not present realistically the variability of landmarks. In this paper, the distribution of a set of landmark points were modeled by non-parametric probability functions $p_{i}\left(\mathbf{x}_{i}\right)$, where $p_{i}(x, y, z)$ is a probability that the landmark $i$ appears at the position $(x, y, z)$, and $\mathbf{x}_{i}=\left(x_{i}, y_{i}, z_{i}\right)$ denotes the 3-D position of the landmark point.

Since the heart contains only a few anatomic landmark points, a set of semi-landmarks were used. In practice, an approximately uniformly distributed set of points $\mathbf{L}$ was chosen from the surfaces of the mean model. The transformations $\mathbf{d}_{i}^{\prime}$ applied to $\mathbf{L}$ define the location of each landmark point in the volumes $V_{i}$. In practice, the probability map of the landmark $i$ is a volume, $W_{i}$, centered around the landmark in the mean volume. The dimensions of $W_{i}$ are large enough to contain the landmark $i$ in all volumes $V_{i}, i \in \Omega$. If the number of the volumes $V_{i}$ were high, the probability values could be accurately estimated by computing the number of landmarks in each voxel and normalizing the 


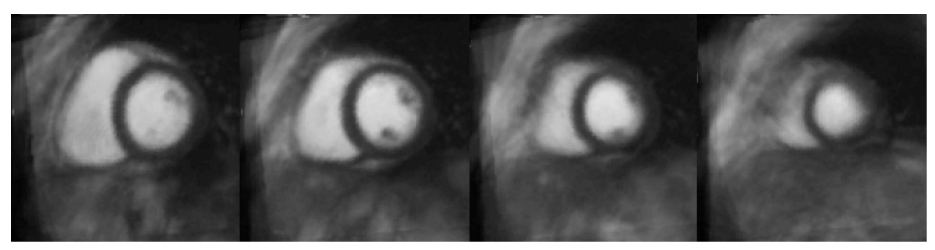

(a)

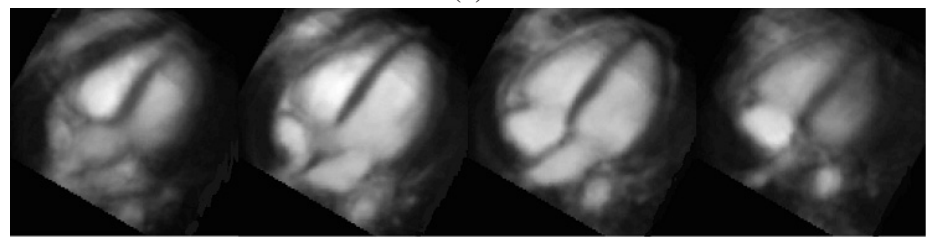

(b)

Fig. 3. Mean a) SA images and b) LA images.

value by the total number of landmarks. Because the number of volumes in our database is relatively low, we used Parzen windowing with a Gaussian kernel $(\sigma=5)$ before the normalization [14].

\section{Results}

In Fig. 3 slices from the mean SA and LA volumes are shown. The images represent a mean in the sense of shape and gray-scale.

Fig. 4 shows the first four modes for SDM. The weight $b_{k}$ for the mode $k$ was $\pm 3 \sqrt{\lambda_{k}}$ where $\lambda_{k}$ is the eigenvalue of the eigenvector $\phi_{k}$ (Eq.2).

Probability distributions for a set of landmark points are shown in Fig. $5 \mathrm{a}$ and b. The figure shows the projection of the 3-D map to the 2-D slice level. We have also implemented a software tool by which a user can study the spatial relationships between landmarks. First, a user indicates a point $\mathbf{q}_{i}$ in the vicinity of one landmark $i$ (white crosses in Fig. 56 and d). The maps show the spatial distribution of all landmark points assuming that the landmark $i$ would be close to the point $\mathbf{q}_{i}$. The landmark is considered to be close to $\mathbf{q}_{i}$ in the tool if its distance to $\mathbf{q}_{i}$ is lower than a user-defined parameter $r$ ( $r \approx 4$ voxels and contained points only from three database subjects in Fig. $5 \mathrm{r}$ and $\mathrm{d}$ ).

\section{Discussion}

In this paper, we described a 3-D statistical shape model of the heart including atrias, ventricles and epicardium. The new approach, that combined information both from standard SA and LA cardiac images, made possible the modeling of the atrias as well as the basal and apical regions of the ventricles.

The shape variations were modeled in two ways: using SDM and using probability distributions. A few deformation modes computed modeled mainly the global shape of the objects. For comparison, we computed also the corresponding modes for active shape 
1st mode
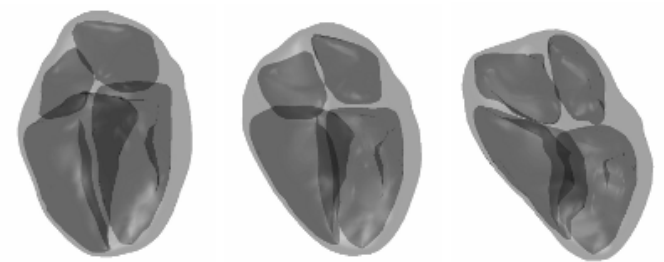

2nd mode
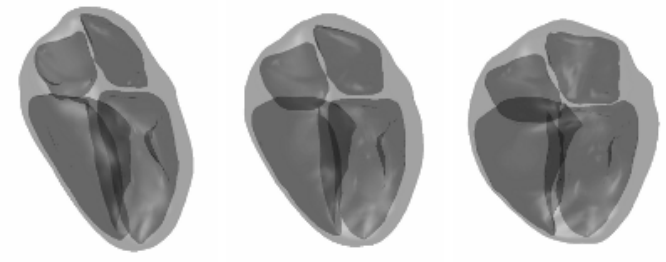

3rd mode
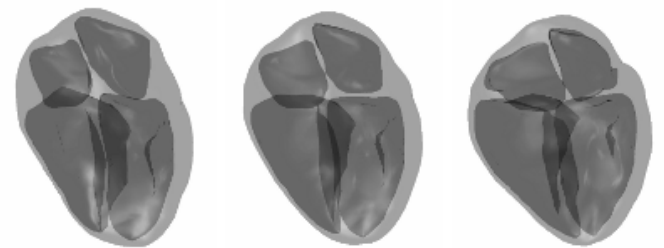

4th mode
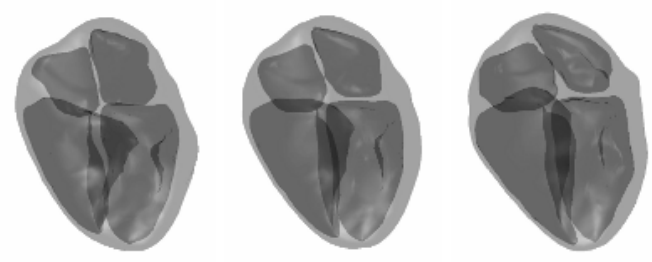

$$
-3 \sqrt{\lambda_{i}}
$$

mean

$$
+3 \sqrt{\lambda_{i}}
$$

Fig. 4. First four deformation modes for SDM.

models. However, no big differences to SDM modes were detected. A new approach of non-parametric probability distributions was also proposed. It can be used to inspect visually the spatial distributions and correlations in shape. However, the database used (16 datasets) was too small for accurate estimation and modeling of shape changes in SDM and in probability maps.

In this study, the datasets were acquired using a standard protocol for cardiac patients. The slice thickness could have been smaller to achieve a better spatial resolution and the SA images could have contained also the atrias to get comprehensive information on atrias. In addition, the dataset could have been volumetric in order to reduce problems from patient movement. Although datasets were not optimal for the model construction, the advantage in using standard sets is, however, that we do not need any specific imaging protocols to increase the size of the database but all acquired datasets in HUCH can be added to the database if no severe movement artifacts are present in images.

In future studies, we will apply the model in cardiac segmentation. The model can be used to regulate the non-rigid transformation using the information on typical shape 


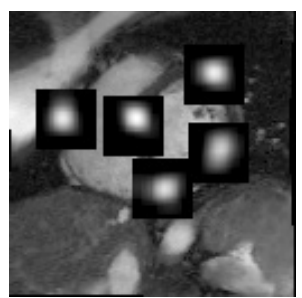

(a)

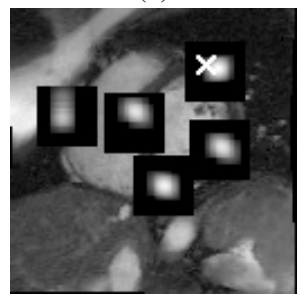

(c)

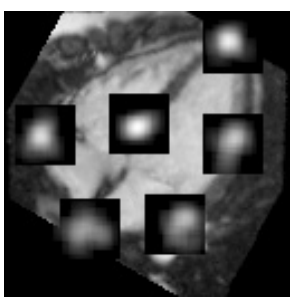

(b)

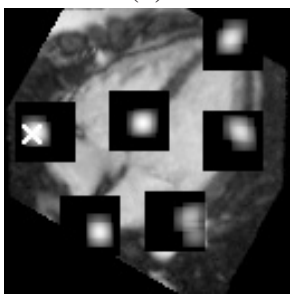

(d)

Fig. 5. Probability distributions shown on an a) SA slice and b) LA slice. Probability distributions considering the landmark would be inside the circle for an c) SA slice and d) LA slice.

changes. In addition, because of small database we could not demonstrate the advantages of the non-parametric approach to the approach using a Gaussian normal distribution. It remains still open if the generalized probability distributions would provide any advantages for the study of shape changes in SA and LA images. Nevertheless, as we extend the database and start to model the heart motion from cine data, we believe that the normal distribution is not anymore general enough in modeling, for example, the twisting motion of the heart.

Acknowledgements. Research was supported by the National Technology Agency, Finland.

\section{References}

1. A.F. Frangi, W.J. Niessen and M.A. Viergever. Three-Dimensional Modeling for Functional Analysis of Cardiac Images: A review. IEEE Trans. Med. Imag., 20(1): 2-25, 2001.

2. P.M. Thompson and A.W. Toga. Detection, Visualization and Animation of Abnormal Anatomic Structure with a Deformable Probabilistic Brain Atlas Based on Random Vector Field Transformation. Med. Image Anal., 1(4): 271-294, 1997.

3. M. Chen, D. Pomerleau and J. Schneider. 3-D Deformbale Registration of Medical Images Using a Statistical Atlas. Lecture Notes in Computer Science 1679: Medical Image Computing and Computer-Assisted Intervention, MICCAI99, Eds. C. Taylor and A. Cochester, pp. 621630, 1999.

4. A. Caunce and C.J. Taylor. Building 3D Sulcal Models using Local Geometry. Med. Image Anal., 5(1): 69-80, 2001. 
5. D.L.G. Hill, J.V. Hajnal, D. Rueckert, S.M. Smith, T. Hartkens and K. McLeish. A Dynamic Brain Atlas. Lecture Notes in Computer Science 2488: Medical Image Computing and Computer-Assisted Intervention - MICCAI 2002, Eds. D. Dohi and R. Kikinis, pp. 532-539, 2002.

6. A.F. Frangi, D. Rueckert, J.A. Schnabel and W.J. Niessen. Automatic Construction of Multiple-Object Three-Dimensional Statistical Shape Models: Applications to Cardiac Modeling. IEEE Trans. Med. Imag., 21(9): 1151-1166, 2002.

7. S.C. Mitchell, J.G. Bosch, B.P.F. Lelieveldt, R.J. van der Geest, J.H.C. Reiber and M. Sonka. 3-D Active Appearance Models: Segmentation of Cardiac MR and Ultrasound Images. IEEE Trans. Med. Imag., 21(9): 1167-1178, 2002.

8. T.F. Cootes, C.J. Taylor, D.H. Cooper and J. Graham. Active Shape Models - Their Training and Application. Computer Vision and Image Understanding, 61(1): 38-59, 1995.

9. T.F. Cootes, G.J. Edwards and C.J. Taylor. Active Appearance Models. Proc. Eur. Conf. Computer Vision, vol. 2, pp. 484-498, 1998.

10. D. Rueckert, A.F. Frangi and J.A. Schnabel. Automatic Construction of 3D Statistical Deformation Models Using Non-rigid Registration. Lecture Notes in Computer Science 2208: Medical Image Computing and Computer-Assisted Intervention - MICCAI 2001, Eds. W.J. Niessen and M.A. Viergever, pp. 77-84, 2001.

11. G.J. Grevera and J.K. Udupa. Shape-Based Interpolation of Multidimensional Grey-Level Images. IEEE Trans. Med. Imag., 15(6): 881-892, 1996.

12. C. Studholme, D.L.G. Hill and D.J. Hawkes. An overlap invariant entropy measure of 3D medical image alignment. Pattern Recognition, 32(1): 71-86, 1999.

13. J. Lötjönen and T. Mäkelä. Elastic matching using a deformation sphere. Lecture Notes in Computer Science 2208: Medical Image Computing and Computer-Assisted InterventionMICCAI 2001, Eds. W.J. Niessen and M.A. Viergever, pp. 541-548, 2001.

14. R.O. Duda and P.E. Hart. Pattern Classification and Scene Analysis, John Wiley \& Sons, 1973. 